\title{
Sistema inalámbrico de adquisición de vibraciones orientado a la detección de movimientos sísmicos con tecnología Zigbee
}

\author{
Reynaldo Claros Lamus \\ MSc(c) de Controles Industriales, \\ Universidad de Pamplona \\ Docente Tiempo Completo, Investigador Grupo UNITEL, \\ Universidad Santo Tomás de Aquino USTA \\ Bucaramanga, Colombia \\ rclaros@hotmail.com
}

\author{
Jaime Andrés Casanova Arenas \\ Ingeniero de Telecomunicaciones, \\ Universidad Santo Tomás de Aquino USTA \\ Auxiliar de Investigación Grupo UNITEL \\ Bucaramanga, Colombia \\ indumil007@hotmail.com
}

\begin{abstract}
Resumen - El proyecto descrito en este trabajo titulado sistema inalámbrico de adquisición de vibraciones orientado a la detección de movimientos sísmicos con tecnología Zigbee, toma como punto de referencia el concepto de la optimización de sistemas de medición de magnitudes físicas y busca principalmente flexibilizar los procesos de monitorización de sismos y análisis de vibraciones con nuevos avances tecnológicos. El trabajo consta de una etapa teórica que explica los fundamentos de cada una de las tecnologías implementadas en el proyecto y una etapa que describe el proceso de diseño y construcción del sistema como tal. El sistema inalámbrico de adquisición de vibraciones propuesto en este trabajo se desarrolló con el fin de lograr un sistema pequeño, eficiente y de bajo costo que tuviera la capacidad de captar, procesar y transmitir la información de las vibraciones registradas alrededor de su medio. Al describir secuencialmente la estructura del proyecto, se comienza con un estudio sobre los acelerómetros como transductores, el cual abarca lo relacionado con los principios básicos de composición, funcionamiento y campos de aplicación, además, se realiza una introducción a la tecnología de sistemas micro electro-mecánicos (MEMS), cuyo papel se describe en el campo de la elaboración de transductores. Otros temas planteados en el proyecto como la energía fotovoltaica y Zigbee, también tienen cabida en el trabajo y hacen parte de la etapa de fundamentos teóricos del proyecto. La etapa de diseño y construcción del sistema propuesto describe al comienzo el proceso de selección del transductor (acelerómetro) a implementar, posteriormente se toma registro de las pruebas realizadas al acelerómetro mediante un análisis de los resultados obtenidos. Seleccionado el transductor se inicia el diseño de la interfaz de adquisición y digitalización de sistema, se describe las etapas que la conforman $y$, a su vez, los componentes que hacen parte de ella. Se describen las principales características del módulo inalámbrico Zigbee implementado así como el proceso de configuración del mismo, además se hace referencia a las etapas de diseño de la interfaz grafica de usuario se caracterizan las partes que la componen y sus respectivas funciones, luego de esto se definen las características funcionales de las celdas fotovoltaicas a implementar y finalmente se toma registro de una serie de pruebas realizadas con el sistema de adquisición de vibraciones totalmente
\end{abstract}

terminado se evalua su funcionalidad y se analiza su desempeño en distintos escenarios donde se presenten sismos o vibraciones.

Palabras clave - Acelerómetro, celdas fotovoltaicas, vibraciones, zigbee.

Abstract - The project described in this paper entitled wireless vibration acquisition system oriented to detect seismic movements implementing ZigBee technology, takes as a reference point the optimization concept of systems for measuring physical quantities and its principal goal is to ease the process of monitoring and analysis of seismic movements and vibrations implementing new technological advances. The work consists of a stage theory that explains the basics of each of the technologies implemented in the project and a phase that describes the process of design and construction of the system itself. The wireless vibration acquisition system proposed in this work was developed in order to achieve a system small, efficient and low cost which would have the ability to capture, process and transmit the information of the vibrations recorded in their environment. Describing the structure of the project sequentially, initially start with a study of the accelerometers as transducers, which include matters related to the basic principles of composition, performance and application fields, we also carried out an introduction to the technology of micro electro-mechanical systems (MEMS), and describe their role in the field of development of transducers. Other issues raised in the project as the principles of photovoltaics and ZigBee, are also accommodated in the workplace are part of the theoretical phase of the project. The stage design and construction of the wireless vibration acquisition system described at the beginning the selection process of the transducer (accelerometer) to implement, after this is taken from the respective recording to the accelerometer tests with analysis of the results. Selected transducer began designing of the interface of acquisition and digitization of the system, describing the stages that compose, and turn the components that are part of it. It describes the main features of the ZigBee wireless modules implemented and the configuration process of the same, also refers to the design stages of the graphical user interface, characterizing the parts which compose it 
and their respective roles, after this is define the functional characteristics of photovoltaic cells to implement, and finally is taken the recording from a series of tests with the wireless vibration acquisition system fully finished evaluating their operation and analyzing its performance in various scenarios where seismic movements occur and vibration.

Keywords - Accelerometer, photovoltaic cells, vibrations, zigbee.

\section{INTRODUCCIÓN}

Los procesos de monitorización de actividad sísmica y exploración geofísica, han sido actividades de constante estudio y análisis durante muchos años, ya que en torno a estos procesos giran factores tan importantes como la prevención de desastres en caso de un terremoto y la detección de hidrocarburos subterráneos. Estos factores, a su vez, se relacionan con los procesos de análisis de vibraciones, que en el área de ingeniería, son una potente herramienta para la detección de imperfecciones en una estructura y fallas en un sistema de maquinaria.

Por estas razones, los procesos de monitorización de sismos y análisis de vibraciones se han convertido en un área de estudio muy importante y en la mayoría de los casos, rentable para los sectores minero, industrial y de ingeniería en general. Con el desarrollo del sistema de adquisición de vibraciones descrito en este trabajo se busca obtener una plataforma versátil en funcionamiento y de gran aplicabilidad especialmente en las áreas de ingeniería y geofísica, con nuevas tecnologías y componentes que, juntos, integran un sistema de diseño no complejo pero robusto en funcionalidad y eficiencia.

\section{FUNDAMENTOS TEÓRICOS}

\section{A. Teoría de los sismos}

Un sismo es un evento físico que se produce cuando hay un desplazamiento de la corteza terrestre, junto con este desplazamiento se libera cierta cantidad de energía la cual se propaga a través del terreno en forma ondas sísmicas. Durante un sismo se producen dos tipos de ondas sísmicas:

1) Ondas de cuerpo: Son perturbaciones que viajan a través del interior de la tierra y siguen caminos curvos debido a la variada densidad y composición de las capas de corteza terrestre. Estas ondas son las primeras en registrarse durante un sismo y generalmente son de baja intensidad. Se pueden identificar dos tipos de ondas de cuerpo, las ondas P y S. Las ondas P son ondas longitudinales que pueden desplazarse a través de medios sólidos o líquidos las cuales se mueven en virtud de la dilatación y compresión de las capas de corteza terrestre o de otro medio que atraviesen. La Fig. 1 [2] muestra la representación de las ondas $\mathrm{P}$ propagándose a través de un medio.

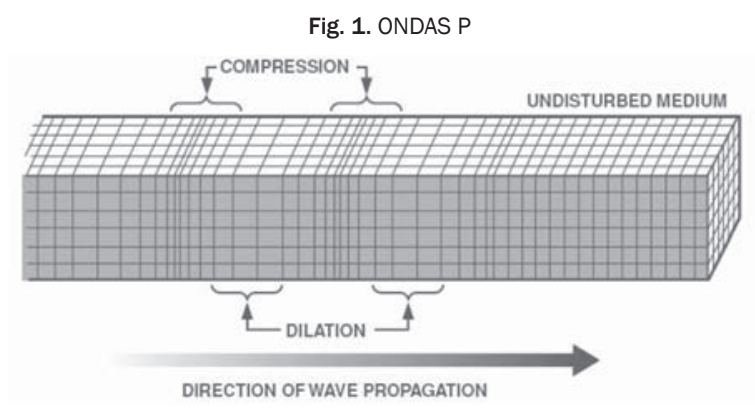

Fuente: Los autores [2]

Las ondas S se desplazan sólo a través de medios sólidos de forma transversal y perpendicular a la dirección de propagación. La Fig. 2 [2] muestra la representación de las ondas $\mathrm{S}$.

Fig. 2. ONDAS S

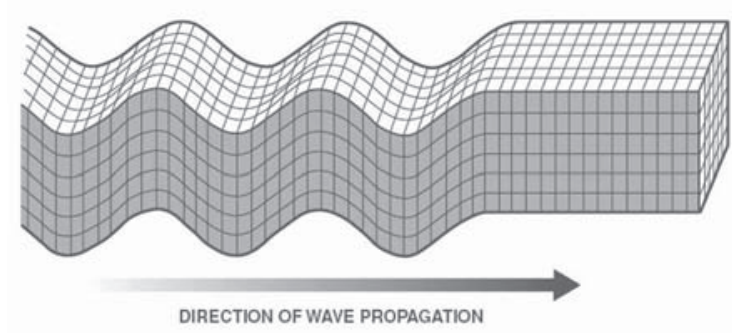

Fuente: Los autores [2]

2) Ondas superficiales: Se propagan sólo por la superficie, desarrollan largos periodos vibratorios y se registran luego de las ondas de cuerpo. Existen dos clases de ondas superficiales, las ondas de Rayleigth, en las cuales el movimiento de las partículas se desarrolla de forma circular y las ondas de Love, en donde el movimiento es horizontal y perpendicular a la dirección de propagación. La Fig. 3 [2] y la Fig. 4 [2] muestran las representaciones de las ondas Rayleigth y las ondas Love respectivamente. 


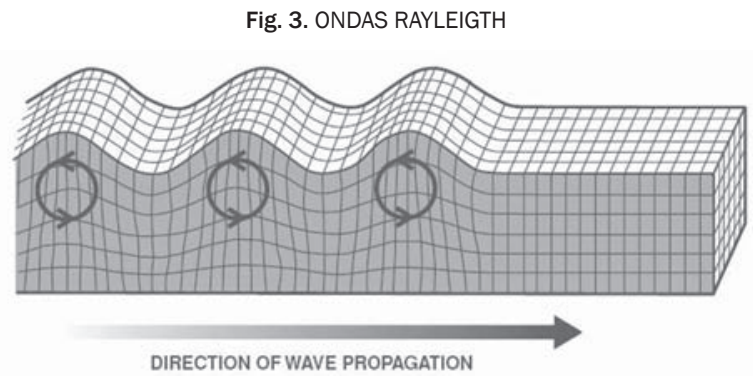

Fuente: Los autores [2]

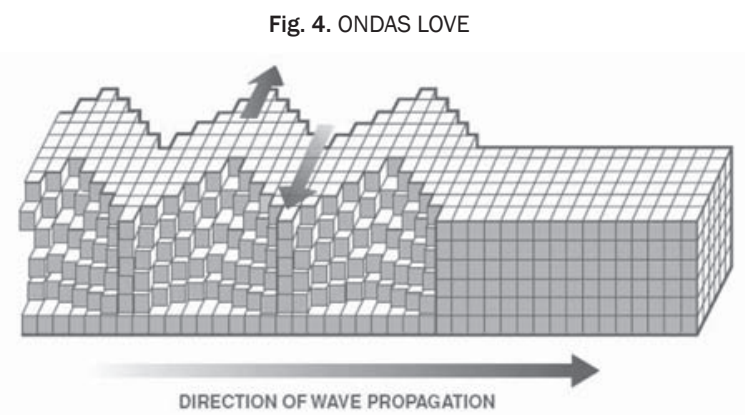

Fuente: Los autores [2]

\section{B. Medición de vibración}

La medición y análisis de vibraciones es una de las herramientas más eficaces en el área de ingeniería ya que por medio de ésta es posible detectar imperfecciones en una estructura y fallas en un sistema de maquinaria. Básicamente se puede definir la medición de vibraciones como el estudio de las oscilaciones mecánicas en un sistema dinámico. Las etapas que conforman un sistema de medición de vibraciones se basan en las etapas básicas de un sistema de adquisición de datos siendo la más relevante la etapa transductora debido a que es la encargada de representar eléctricamente las vibraciones captadas. Los transductores empleados en los procesos de medición de vibraciones producen una señal eléctrica la cual es proporcional a la magnitud medida y generalmente son utilizados para medir la aceleración, velocidad y desplazamiento en un sistema sometido a vibraciones. Existen diferentes tipos de transductores de vibración entre los que podemos encontrar los acelerómetros y los transformadores diferenciales variables.

\section{COMPOSICIÓN DEL SISTEMA PROPUESTO}

El sistema inalámbrico de adquisición de vibraciones tiene la capacidad de captar, procesar, trans- mitir y presentar la información de las vibraciones registradas alrededor de su medio. Para lograr esto el sistema fue diseñado por bloques, cada uno con funciones y características diferentes. La Fig. 5 muestra los bloques que componen el sistema propuesto:

Fig. 5. DIAGRAMA DE BLOQUES DEL SISTEMA PROPUESTO

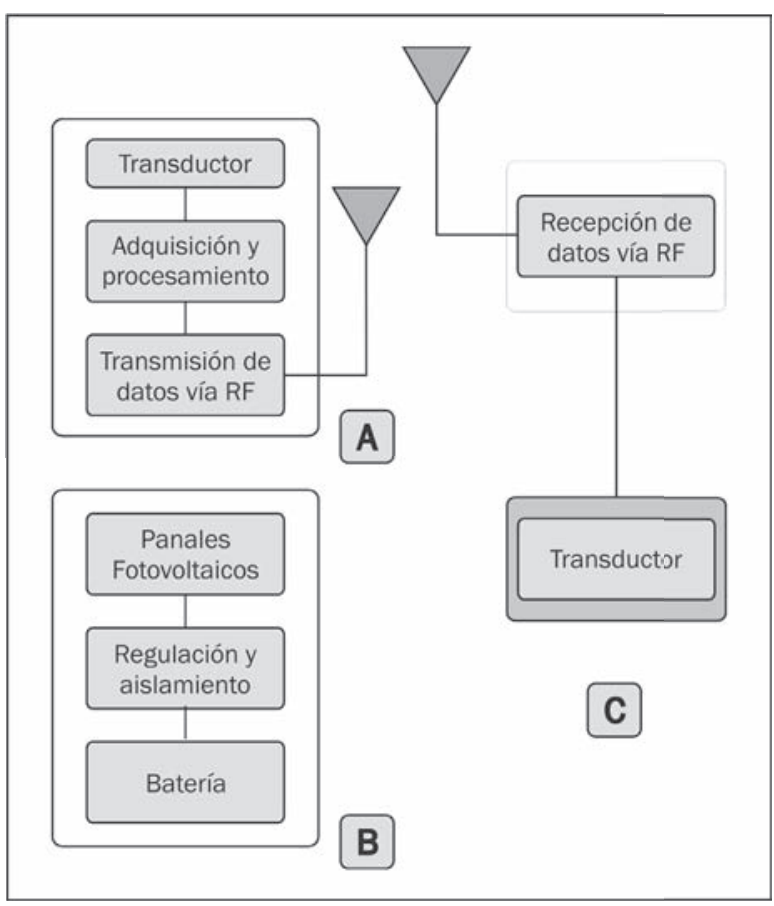

Fuente: Los autores

\section{A. Subsistema electrónico}

Es el encargado de la mayoría de los procesos realizados por el sistema inalámbrico de adquisición de vibraciones y está compuesto por las siguientes etapas:

- Etapa transductora: con el fin de medir las vibraciones registradas en un medio se implementó como transductor del sistema el acelerómetro MMA7260QT, basado en la tecnología de sistemas micro electro-mecánicos (MEMS). Este dispositivo convierte la aceleración de la gravedad o de un movimiento en una señal eléctrica analógica proporcional a la vibración o aceleración a la cual está sometido. El MMA7260QT mide aceleración y vibración en los tres ejes cartesianos con la posibilidad de realizar estas mediciones en 4 rangos distintos de nivel de aceleración y sensibilidad que permiten al dispositivo funcionar en $1.5 \mathrm{~g}, 2 \mathrm{~g}, 4$ g, 6 g. Muchas aplicaciones relacionadas con 
la medición de vibraciones se realizan en estos rangos como la estabilización de motores realizada en $6 \mathrm{~g}$, otros procesos como monitorización sísmica se realizan bajo rangos de medición de 1.5 g y 2 g.

Para verificar el funcionamiento del dispositivo se realizaron una serie de pruebas que tenían como objetivo la adquisición de señales del acelerómetro bajo diferentes rangos de medición de aceleración. La Fig. 6 muestra las señales adquiridas para un rango de aceleraciones de $1.5 \mathrm{~g}$.

Fig. 6. SEÑALES ADQUIRIDAS PARA UN RANGO DE $1.5 \mathrm{G}$

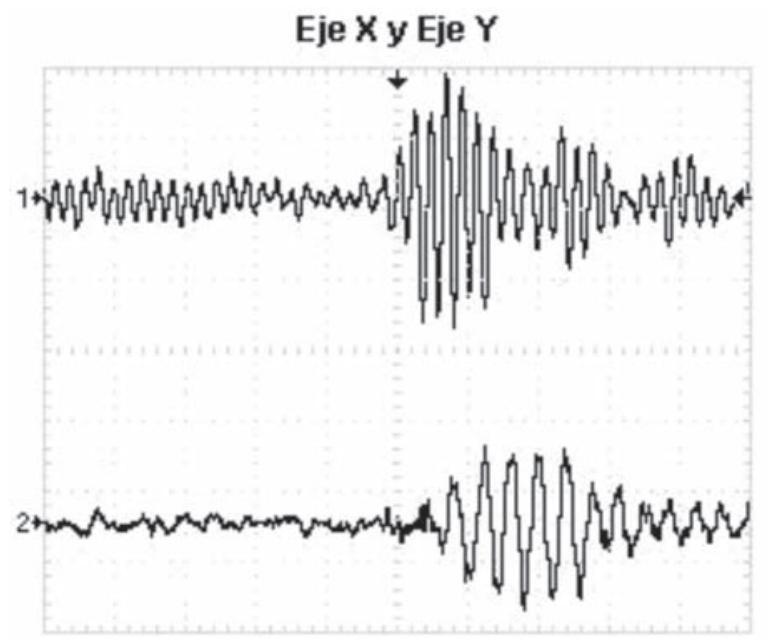

Eje $X$ y Eje $Z$

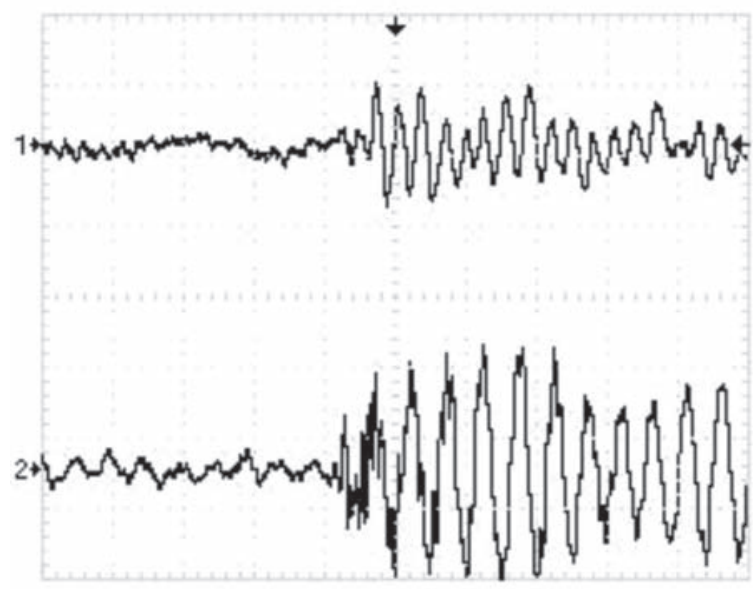

Fuente: Los autores

- Interfaz electrónica de adquisición y procesamiento: Esta interfaz electrónica permite adquirir y convertir las señales eléctricas pro- porcionales a las aceleraciones a las que está sometido el transductor, en datos digitales; para poder transmitirlos inalámbricamente y posteriormente presentarlos a un usuario final.

La interfaz electrónica de adquisición y procesamiento está compuesta principalmente por un conversor análogo/digital externo de arquitectura SAR (Registro de Aproximaciones Sucesivas), de 12 bits de resolución y con múltiples canales de entrada programables, y por el microcontrolador PIC16F648A fabricado por Microchip Technology.

Estos dos componentes se encargan de manera conjunta de la digitalización y procesado de las señales analógicas provenientes del acelerómetro MMA7260QT. Posterior al muestreo de las señales del acelerómetro, se empaquetan los datos resultantes de cada conversión y se transmiten seriamente vía RS232 al modulo de radiofrecuencia encargado de transmitir los datos inalámbricamente.

- Etapa de transmisión y recepción inalámbrica de datos: una vez que el microcontrolador transmite serialmente los datos de las conversiones análoga/digital, se implementó un tipo de módulos de radiofrecuencia embebido conocido como módulos XBee-PRO ZNet 2.5 fabricados por DIGI International, estos módulos soportan el estándar IEEE 802.15.4 en el cual se basa la tecnología Zigbee.

Los módulos XBee-PRO ZNet 2.5 se implementan con el fin de transmitir inalámbricamente los datos provenientes de la interfaz electrónica de adquisición y digitalización a un receptor remoto que estará conectado a un computador que a su vez soportará un software que permitirá la visualización de cada uno de los eventos sísmicos registrados por el sistema de adquisición de vibraciones, el receptor remoto utilizado será al igual que el transmisor, un módulo XBee-PRO ZNet 2.5.

Este tipo de módulos permiten implementar distintas topologías de comunicación entre las que se encuentran las topologías estrella, árbol y malla (ver Fig. 7) especificadas por el estándar IEEE 802.15.4, en las bandas de 2.4 GHz y $900 \mathrm{MHz}$, además, da la posibilidad de trasmitir a distancias considerables, hasta 1.6 
$\mathrm{km}$ con un reducido consumo de energía. Una red basada en dispositivos Zigbee puede estar compuesta por un máximo de 65535 nodos, que permite implementar con estos dispositivos redes de gran densidad.

Fig. 7. TOPOLOGÍAS EXISTENTES EN ZIGBEE

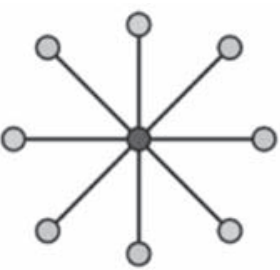

Topologia Estrella

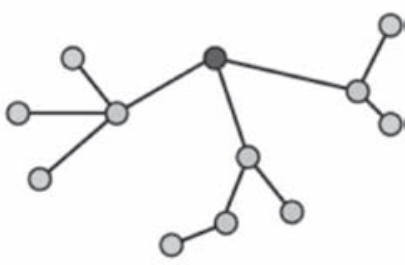

Topologia Arbol

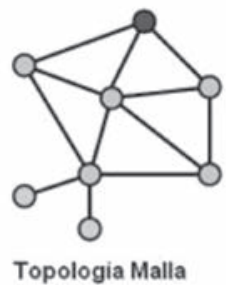

Fuente: Los autores

Debido a que en el sistema propuesto era necesario que existiese una conexión transparente entre un módulo transmisor y un módulo receptor remoto, se implementó una conexión entre módulos XBee-PRO ZNet 2.5 en modo unicast (punto a punto) en la banda de los $2.4 \mathrm{GHz}$.

Una de las ventajas del modo unicast es ser el único modo que soporta reenvío de información, ya que en este, el módulo receptor envía un paquete de confirmación (ACK) al módulo transmisor indicando que la información ha sido recibida correctamente. La Fig. 8 muestra el modelo de conexión implementado con los módulos XBee.

Fig. 8. MODELO DE CONEXIÓN IMPLEMENTADO CON LOS MÓDULOS RF

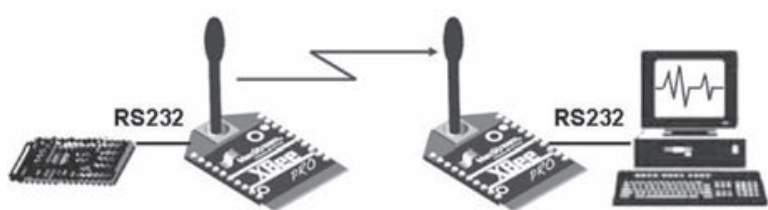

Fuente: Los autores

\section{B. Etapa de alimentación y suministro de energía}

Debido a que el sistema inalámbrico de adquisición de vibraciones fue diseñado para trabajar en diferentes escenarios, muchos de ellos remotos y privados de fuentes de energía constante, la etapa de alimentación del sistema propuesto implementará una fuente de energía portable como lo es una batería con soporte de carga por medio de paneles fotovoltaicos. Sin embargo, debido a que en los sistemas fotovoltaicos que usan baterías pueden presentarse sobrecargas de voltaje se implementó un dispositivo electrónico conocido como regulador paralelo, este dispositivo, además de regular el voltaje de carga proveerá un aislamiento entre los paneles y la batería. La Fig. 8 muestra la configuración básica de un regulador paralelo.

Fig. 9. REGULADOR PARALELO

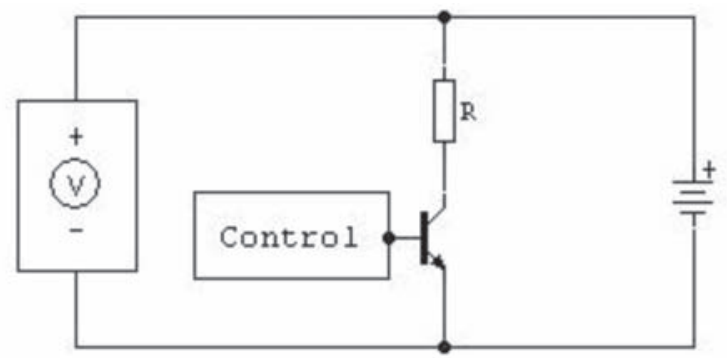

Fuente: Los autores

\section{Software de visualización de señales}

Este software de visualización se realizó en un entorno de desarrollo flexible y altamente funcional, el cual suministró las distintas herramientas para la elaboración del software. El software descrito proveerá al usuario una interfaz gráfica, práctica y eficiente que permitirá la visualización de las señales proporcionales a la vibración aplicada al sistema inalámbrico de adquisición de vibraciones, que facilita el análisis de este tipo de ondas.

La interfaz descrita proveerá una serie de controles y objetos con funciones específicas entre los que están, una casilla que permite el escaneo y selección de puerto COM que será utilizado para la recepción de datos seriales a través de la interfaz electrónica de recepción de datos conectada al computador, una casi- 
Ila de establecimiento de la conexión con el puerto para habilitar la recepción de datos, tres despliegues gráficos correspondientes a cada eje de medición cartesiano en los cuales se graficarán las señales proporcionales a las vibraciones registradas alrededor del sistema propuesto, tres botones deslizables que permiten ajustar la visualización de las señales presentadas en el eje tiempo y una barra de desplazamiento horizontal que permite definir el punto de origen desde el cual se graficarán las señales.

La Fig. 10 muestra la interfaz gráfica de usuario funcionando, se puede apreciar varias vibraciones registradas con distinta intensidad y frecuencia.

Fig. 10. INTERFAZ GRÁFICA DE USUARIO

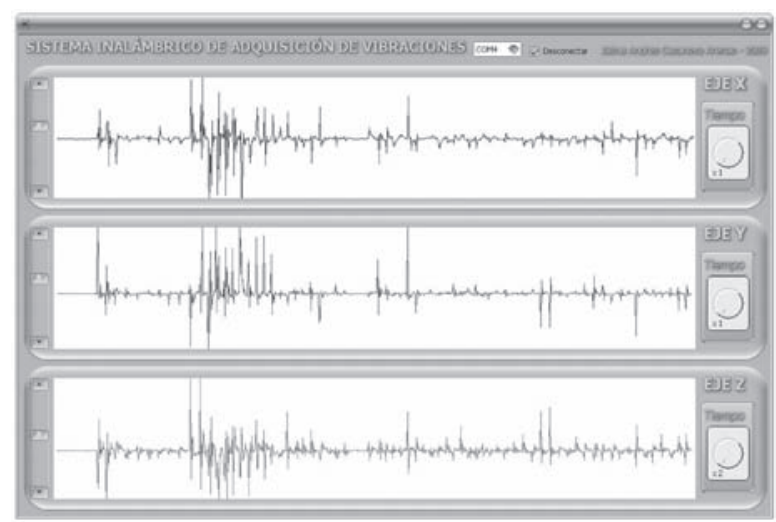

Fuente: Los autores

\section{PRUEBAS Y RESULTADOS OBTENIDOS}

Con el fin de evaluar el funcionamiento del sistema inalámbrico de adquisición de vibraciones totalmente terminado se realizaron una serie de pruebas las cuales consistieron en someter el sistema propuesto a diferentes fuentes de vibraciones en diferentes lugares, con el fin de visualizar las distintas señales generadas por las vibraciones, analizando especialmente la intensidad y la frecuencia con que éstas se registran que depende del tipo de fuente vibratoria al que fue sometido el sistema y, de esta manera, realizar la validación de los datos adquiridos.

La Fig. 11 presenta el registro de un evento vibratorio producido en un instante específi- co, con esta prueba se verifica el tiempo de respuesta del sistema propuesto al registrarse una vibración.

Fig. 11. REGISTRO DE UNA VIBRACIÓN GENERADA EN UN INSTANTE ESPECÍFICO

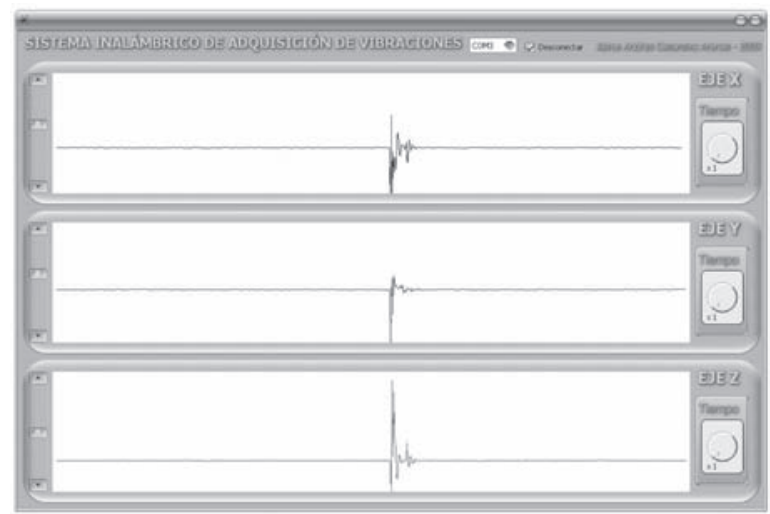

Fuente: Los autores

La Fig. 12 muestra el registro de impactos de media y alta intensidad generados con un objeto contundente a cierta distancia del dispositivo. Con esta prueba se confirma el correcto funcionamiento del sistema ante la presencia de impactos producidos en forma aleatoria.

Fig. 12. REGISTRO DE IMPACTOS ALEATORIOS DE MEDIA Y ALTA INTENSIDAD

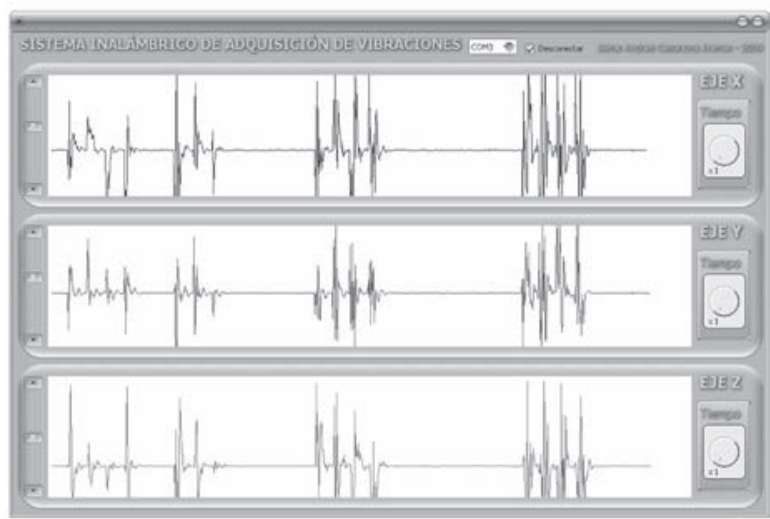

Fuente: Los autores

La Fig. 13 muestra el registro de vibraciones de baja intensidad, con esta prueba se evaluó la sensibilidad del sistema propuesto y su capacidad de registrar eventos vibratorios de pequeña intensidad. 
Fig. 13. REGISTRO DE VIBRACIONES DE BAJA INTENSIDAD

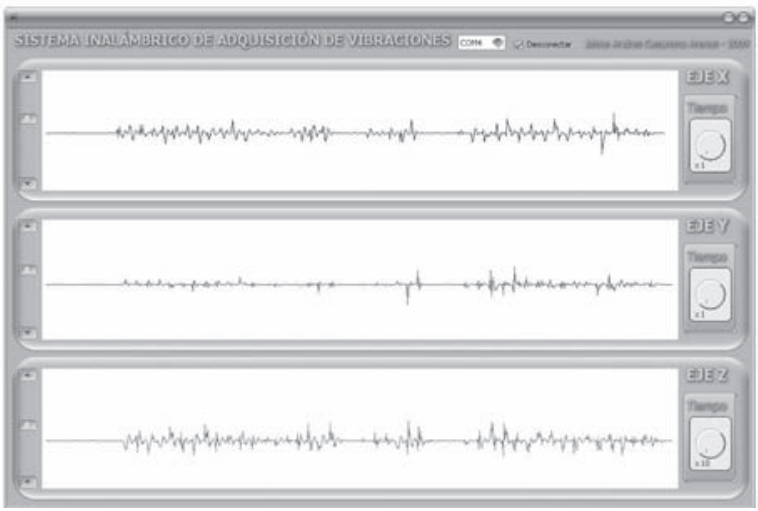

Fuente: Los autores

En la Fig. 14 se muestra la ubicación del sistema propuesto para registrar las vibraciones generadas en un escenario exterior.

La Fig. 15 presenta el registro de las vibraciones generadas por el paso de vehículos sobre una carretera, en este registro se logra apreciar gran cantidad de eventos vibratorios de diferente intensidad y frecuencia.

Esta prueba se realizó con el fin de evaluar el funcionamiento del sistema en un escenario exterior en donde se registrarán diversos tipos de vibraciones.

Fig. 14. PRUEBA REALIZADA PARA REGISTRAR VIBRACIONES EN UN ESCENARIO EXTERIOR.

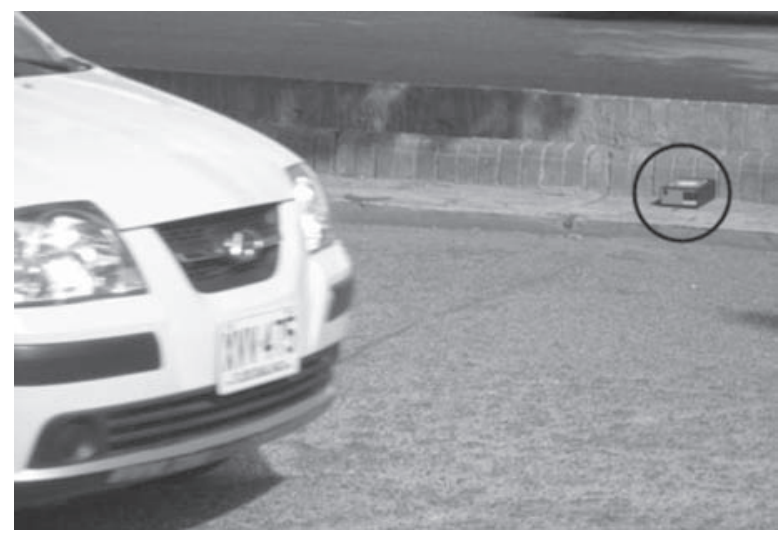

Fuente: Los autores
Fig. 15. REGISTRO DE LAS VIBRACIONES GENERADAS POR EL PASO DE VEHÍCULOS.

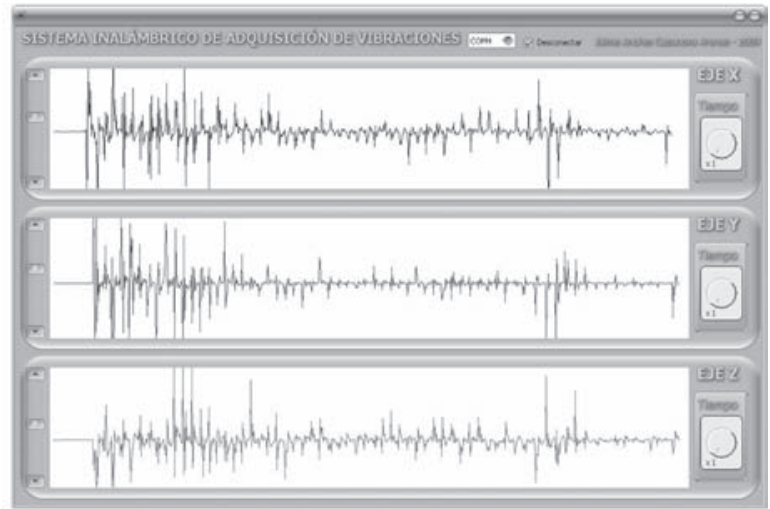

Fuente: Los autores

Luego de realizadas varias pruebas, incluidas las anteriormente descritas, se pudo constatar el óptimo funcionamiento del sistema inalámbrico de adquisición de vibraciones en diferentes escenarios en los cuales se puedan registrar vibraciones. La Fig. 16 muestra el sistema propuesto en su totalidad.

Fig. 16. SISTEMA INALÁMBRICO DE ADQUISICIÓN DE VIBRACIONES

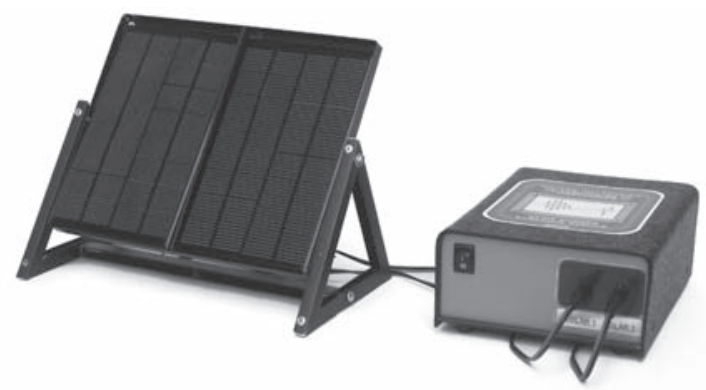

Fuente: Los autores

La Fig. 17 y la Fig. 18 muestran el interior del sistema inalámbrico de adquisición de vibraciones observándose todos los bloques funcionales que lo componen. 
Fig. 17. VISTA LATERAL DEL INTERIOR DEL SISTEMA INALÁMBRICO DE ADQUISICIÓN DE VIBRACIONES.

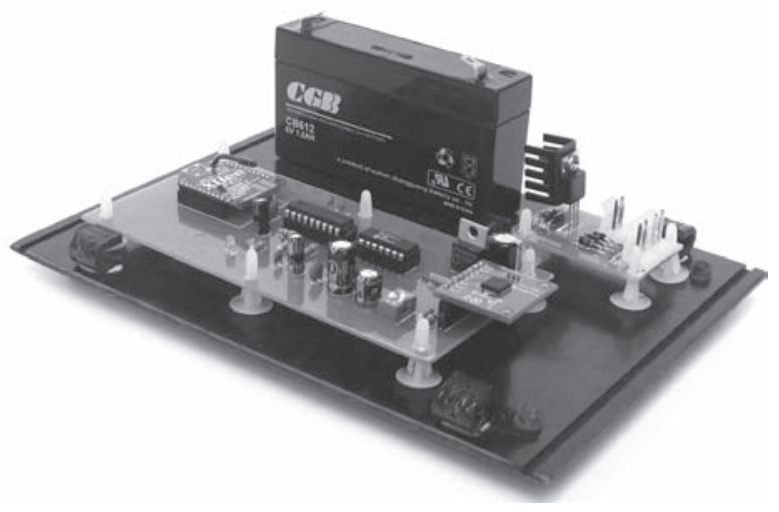

Fuente: Los autores

Fig. 18. VISTA SUPERIOR DEL INTERIOR DEL SISTEMA INALÁMBRICO DE ADQUISICIÓN DE VIBRACIONES

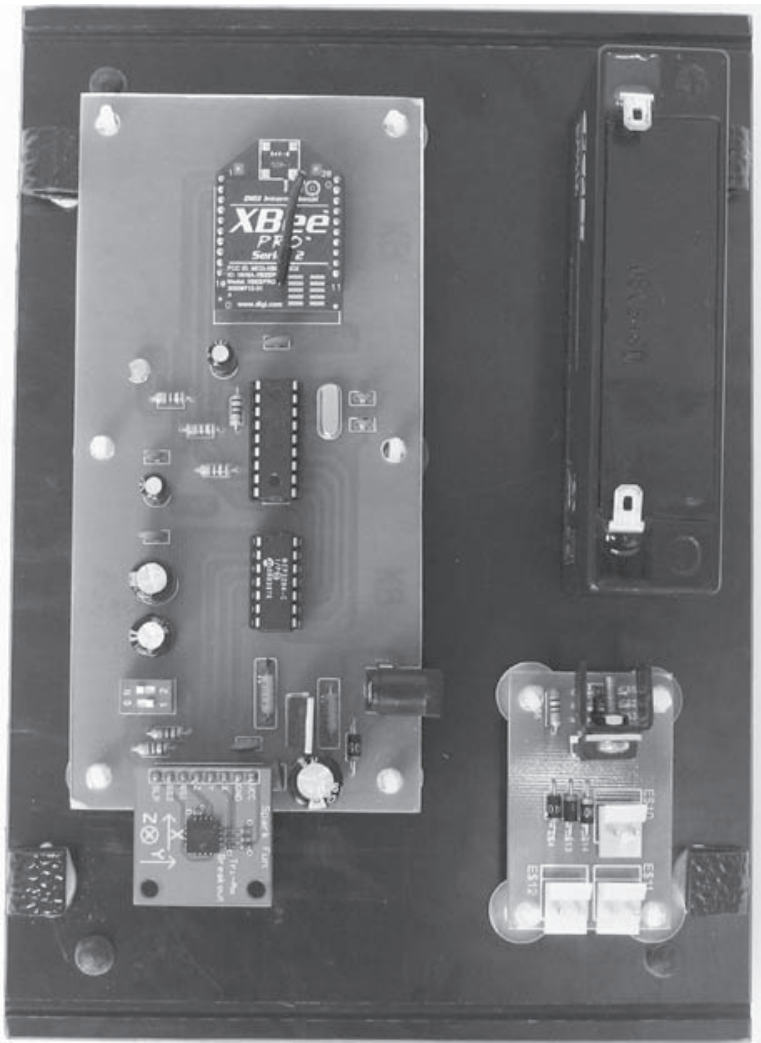

Fuente: Los autores

\section{CONCLUSIONES}

Muchas ventajas y funcionalidades que provee el sistema inalámbrico de adquisición de vibraciones se deben a que éste implementa nuevas tecnologías en el campo de los transductores y módulos inalámbricos embebidos como también en el campo de las energías alternativas, convirtiéndolo en un sistema innovador con amplio rango de aplicación.

La interfaz electrónica de adquisición y procesamiento del sistema inalámbrico de adquisición de vibraciones permite registrar de manera rápida y confiable los eventos sísmicos y las vibraciones que se presenten en diversos escenarios, esto debido en gran parte a la eficiencia en los procesos de conversión analógica a digital y en el procesado de los datos digitalizados, soportados por la tecnología Flash y la arquitectura SAR en las que están basados respectivamente el microcontrolador PIC y el conversor análogo/digital.

La implementación de los módulos inalámbricos XBee-PRO ZNet 2.5 en la etapa de transmisión y recepción de datos del sistema inalámbrico de adquisición de vibraciones, permitió realizar una comunicación inalámbrica de forma rápida, robusta y eficiente

El software de visualización de señales del sistema inalámbrico de adquisición de vibraciones provee al usuario final una interfaz gráfica, amigable y con variedad de herramientas para el análisis intuitivo de los registros correspondientes a los eventos vibratorios.

Este sistema inalámbrico de adquisición de vibraciones es una alternativa práctica y eficiente que puede utilizarse en técnicas de diagnóstico de fallas y evaluación de la integridad de estructuras y máquinas.

\section{REFERENCIAS}

[1] MAQ, ANÁLISIS DE MAQUINARIA. Tutorial De Vibraciones Para Mantenimiento Mecánico [Archivo PDF]. Enero del 2005. Disponible en: http://www.a-maq.com

[2] ANALOG DEVICES. Sensing, Analyzing, and Acting in the First Moments of an Earthquake [Archivo PDF]. 
Analog Dialogue 35-1. Disponible en: http://www. analog.com/library/analogDialogue/archives/35 01/ earthquake/earthquake.pdf

[3] LUQUE ESTEPA, Antonio. Diseño de un acelerómetro basado en tecnología MEMS (I) [Archivo PDF]. Dpto. Ingeniería Electrónica. Disponible en Internet <URL: www.gte.us.es/ASIGN/SEA/MEMS_PRACT1.pdf >

[4] ORTEGA HUEMBES, Carlos Alberto, DEL SOCORRO ROQUE, Deyanira y ÚBEDA SEQUEIRO, Leslie Eduardo. Zigbee: El nuevo estándar global para la Domótica e Inmótica [Archivo PDF]. Universidad Nacional de Ingeniería, Facultad de Electrotecnia y Computación [Managua, Nicaragua]: 28 de julio de 2008.

[5] PERNIA MÁRQUEZ, Daniel. INTRODUCCIÓN A LA MEDICIÓN DE VIBRACIÓN [Archivo PDF]. Universidad de los Andes. Facultad de Ingeniería. [Mérida, Venezuela]: 03 de Febrero de 2004.

[6] PRATO, Carlos A. y FLORES, Fernando. ACCIONES SISMICAS PARA DISEÑO ESTRUCTURAL [Archivo PDF]. Cátedra de Análisis Estructural: 2007. Disponible en Internet: <URL: http://www.efn.uncor.edu/departamentos/estruct/ana_est_ic/Acciones_Sismicas.pdf >

[7] RUIZ FERNÁNDEZ, Daniel y MORENO, Javier Martín. Informe Técnico: Protocolo ZigBee (IEEE 802.15.4) [Archivo PDF]. Universidad de Alicante [Alicante, España]: Junio de 2007.

[8] VIGNA, Benedetto. Physical Sensors Drive MEMS Conzumerization Wave. [Archivo PDF]. ST Microelectronics, MEMS Product Division. Disponible en Internet: <URL: http://www.st.com/stonline/products/families/sensors/motion_sensors/related_info/mmc_japan.pdf>

[9] A. de la Villa y A. Gómez, "Estimadores de estado generalizados de sistemas eléctricos de potencia”, Ingeniería Energética y Medioambiental, año XXXI, n. ${ }^{\circ} 186$, pp. 64-69, jul./ago. 2005.

[10] M. Monard e J. Baranauskas, Conceitos sobre aprendizado de máquinas em sistemas inteligentes: Fundamentos e aplicações, Cap. 4, Tamboré-Barueri, Brasil, Editora Manole, 2003. 